\title{
Imaging photoplethysmography for clinical assessment of cutaneous microcirculation at two different depths
}

Zbignevs Marcinkevics

Uldis Rubins

Janis Zaharans

Aleksejs Miscuks

Evelina Urtane

Liga Ozolina-Moll 


\title{
Imaging photoplethysmography for clinical assessment of cutaneous microcirculation at two different depths
}

\author{
Zbignevs Marcinkevics, ${ }^{\mathrm{a}, \star}$ Uldis Rubins, ${ }^{\mathrm{b}}$ Janis Zaharans, ${ }^{\mathrm{b}}$ Aleksejs Miscuks, ${ }^{\mathrm{c}}$ Evelina Urtane, ${ }^{\mathrm{a}}$ and \\ Liga Ozolina-Molla \\ aniversity of Latvia, Department of Human and Animal Physiology, Faculty of Biology, Raina Boulevard 19, Riga LV-1586, Latvia \\ bUniversity of Latvia, Institute of Atomic Physics and Spectroscopy, Skunu Street 4, Riga LV-1586, Latvia \\ 'Hospital of Traumatology and Orthopaedics, Duntes Street 29, Riga LV-1005, Latvia
}

\begin{abstract}
The feasibility of bispectral imaging photoplethysmography (iPPG) system for clinical assessment of cutaneous microcirculation at two different depths is proposed. The iPPG system has been developed and evaluated for in vivo conditions during various tests: (1) topical application of vasodilatory liniment on the skin, (2) skin local heating, (3) arterial occlusion, and (4) regional anesthesia. The device has been validated by the measurements of a laser Doppler imager (LDI) as a reference. The hardware comprises four bispectral light sources (530 and $810 \mathrm{~nm}$ ) for uniform illumination of skin, video camera, and the control unit for triggering of the system. The PPG signals were calculated and the changes of perfusion index (PI) were obtained during the tests. The results showed convincing correlations for PI obtained by $\mathrm{PPPG}_{530 \mathrm{~nm}}$ and LDI at (1) topical liniment $(r=0.98)$ and (2) heating $(r=0.98)$ tests. The topical liniment and local heating tests revealed good selectivity of the system for superficial microcirculation monitoring. It is confirmed that the iPPG system could be used for assessment of cutaneous perfusion at two different depths, morphologically and functionally different vascular networks, and thus utilized in clinics as a cost-effective alternative to the LDI. ๑2016 Society of Photo-Optical Instrumentation Engineers (SPIE) [DOI: 10.1117/1.JBO.21.3.035005]
\end{abstract}

Keywords: imaging photoplethysmography; cutaneous perfusion; local anesthesia; local heating test; topical application of nicotinates.

Paper 150589RRR received Sep. 9, 2015; accepted for publication Mar. 3, 2016; published online Mar. 30, 2016.

\section{Introduction}

In recent years, there has been a growing demand in cost-effective and contactless reliable techniques for assessment of cutaneous microcirculation for both small family medicine offices and large hospitals. ${ }^{1}$ Assessment of microcirculation is of vital importance and provides essential information for the diagnosis of diseases because cutaneous the neuro-immunoendocrine system plays an important role in controlling physiological functions of the body, ${ }^{2}$ serving as an early marker for chronic diseases, such as diabetes mellitus, Raynaud syndrome, metabolic syndrome, and even neuropathy with the potential application, ranging from routine testing of endothelial function to local anesthesia monitoring. ${ }^{3,4}$ To reduce the influence of measuring procedures, a nonintrusive approach is essential. Over about the last four decades, a variety of contactless photonic methods have been developed and utilized for registering cutaneous circulation, such as laser Doppler flowmetry (LDF), laser speckle contrast imaging (LSCI), ${ }^{6,7}$ and tissue viability imaging (TiVi). ${ }^{8}$ However, all are relatively expensive and have several limitations. The conventional LDF has relatively low scanning speed. LSCI has both high spatial and temporal resolution, but limited penetration depth as a strong scattering of tissue reduces the image contrast, which in turn decreases the sensitivity to flow velocity. ${ }^{9-12} \mathrm{TiVi}$ measures red blood cell

*Address all correspondence to: Zbignevs Marcinkevics, E-mail: zbigis@latnet concentration rather than blood perfusion. The substantial limitation of the aforementioned techniques is the inability to register perfusion from microcirculation layers at different depths. In fact, there were attempts to overcome this limitation, for instance, by separating LDF bandwidths, ${ }^{13}$ but the validity of this method is based on the theoretical assumption that blood flow is lower is superficial layers because of the smaller cross-sectional area and lower flow velocity in capillaries. The interpretation of cutaneous perfusion data registered optically is rather challenging and possibly contradictory due to the nonhomogenous and complex architecture of skin and in particular, cutaneous vasculature, being a heterogeneous medium for the transport of light, with a difficulty to completely reproduce in the phantoms. However, recently, there have been several successful attempts to design complex multilayer skin phantoms for photon penetration studies. ${ }^{14-18}$ A relatively new and promising technique for assessment of cutaneous microcirculation is imaging photoplethysmography (iPPG). ${ }^{19-21}$ It is based on detection of subtle skin color changes in the sequence of video frames and requires an inexpensive consumer range camera and conventional light source. ${ }^{22}$ The key to this technique is the different pixel intensity detection algorithms and computational methods, such as color magnification technique. ${ }^{23,24}$ Previous studies confirmed the potential of iPPG technique in recording of cutaneous perfusion at different depths by means of different wavelength light sources, mostly

$1083-3668 / 2016 / \$ 25.00$ @ 2016 SPIE 
utilizing on-bench tests. ${ }^{25}$ However, to deliver the technology to clinicians, more extensive examination is needed, particularly, in the light of the recently proposed paradigm of iPPG signal genesis. ${ }^{26}$ Therefore, this paper proposes a design and evaluation of bispectral imaging PPG device prototype for clinical monitoring of superficial and deep cutaneous microcirculation during various provocation tests.

\section{Methods}

\subsection{Design of Imaging Photoplethysmography Hardware}

The custom-made iPPG prototype device has been elaborated. To acquire blood volume changes in the deep cutaneous tissue, the reflection mode remote PPG is combined with orthogonal polarization spectral imaging and bispectral light sources, exploiting the principle of different wavelength penetration into different depths of the skin. ${ }^{25,27}$

The custom-made iPPG device has been designed using CAD software (Solidworks, Dassault Systems). The plastic parts were printed with a three-dimensional (3-D) printer (Prusa i3, Custom made, Latvia). The device is composed of a plastic enclosure filled with an adjustable vacuum pillow $(40 \times 20 \mathrm{AB}$ Germa, Sweden $)$ as palm support, four bispectral light sources, monochromatic video camera, and microcontroller board (Arduino Nano, Arduino) (Fig. 1).

The illuminating system is composed of four bispectral light sources, each consisting of two high-power light-emitting diode (LED) emitters (Roithner LaserTechnik GmbH, green: $\lambda=530 \mathrm{~nm}, 3 \mathrm{~W}$; infrared (IR): $\lambda=810 \mathrm{~nm}, 1 \mathrm{~W}$ ) [Fig. 1(a)]. The designed system was intended mostly for recording signals from the curved surface of the hand (dorsal or ventral aspect), and so achieving a uniform illumination can be difficult. To achieve uniform illumination of the skin surface, an adjustable LED intensity control was introduced via PC-based custom developed software. The wavelengths of LEDs were chosen by taking into account the light-tissue penetration depth and isosbestic wavelength of oxyhemoglobin and deoxyhemoglobin to avoid the effect from saturation changes $\left(\mathrm{SpO}_{2}\right)$ as described by Sandberg et al. ${ }^{28}$ The microcontroller board provides switching of green and IR LEDs intermittently and sequential triggering of video frames captured by a camera. The camera control was performed by uEye software using the manual trigger mode, fixed exposure time, $2 \times 2$ pixel binning, and triggered at 60 frames/s. The camera (8 bit CMOS "IDS-uEye UI1221LE") was equipped with "S-mount" $1 / 2$ in. $F=4 \mathrm{~mm}$ low distortion, wide-field lens (Lensagon). For the full view of an adult palm $(20 \times 15 \mathrm{~cm}$ field of view), the camera lens was placed at $15 \mathrm{~cm}$ distance from the skin surface. In order to reduce skin specular reflectance, orthogonally oriented polarizers were placed behind the camera and light sources.

\subsection{Software}

In order to extract hemodynamic information from two different depths of the skin, the sequence of video frames was stored as a video format file and further analysis was carried out offline using custom developed MATLAB-based software (University of Latvia, Uldis Rubins) [Fig. 2(a)]. The following algorithm has been implemented in the software [Fig. 2(b)]: (1) the video frames were downsampled, by a factor of 4 , by averaging neighboring pixels, for processing speed; (2) PPG signals were calculated from the averaged pixels in the selected region of interest (RoI) of video frames; (3) fast varying PPG (AC) component regarding cardiac activity was calculated [using bandpass $(0.7$ to $1.5 \mathrm{~Hz})$ second-order Butterworth filter] and slow (DC) component was calculated [by lowpass (0 to $0.1 \mathrm{~Hz}$ ) second-order Butterworth filter]; and (4) the hemodynamic parameters [pulse rate, PPG amplitude, and perfusion index (PI)] were calculated from the PPG AC and DC signals. The last step involved storage of calculated hemodynamic data accessible for third-party software, such as SigmaPlot.

The software allows manual selection of the RoI and time window of video. The hemodynamic parameters are calculated

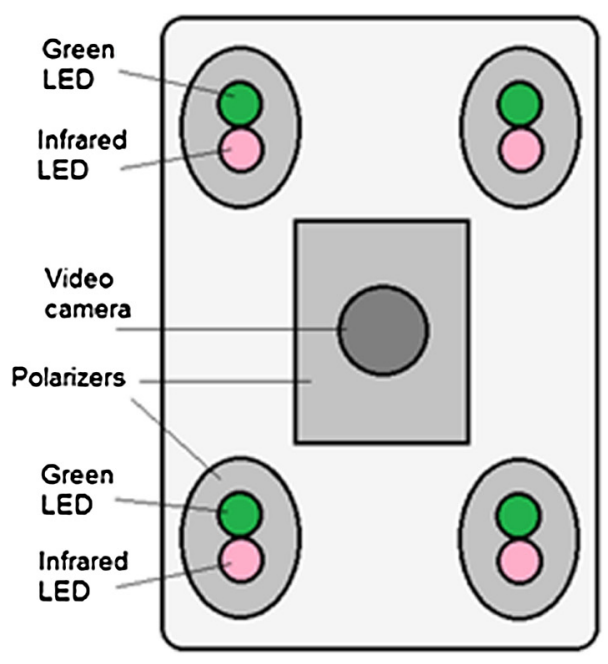

(a)

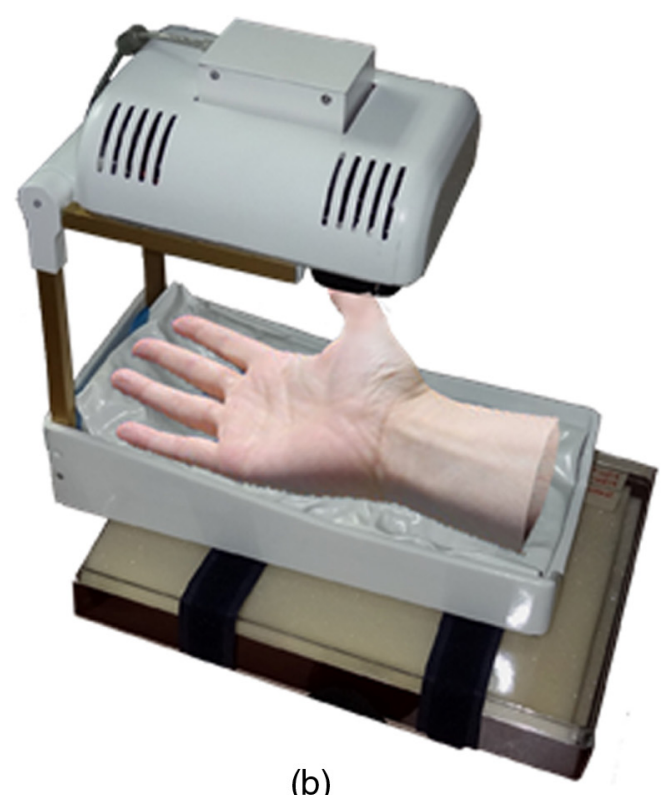

(b)

Fig. 1 (a) Hardware components of imaging PPG device: bottom view of imaging system, camera, and light sources and (b) the device fastened to the basement, including the body with vacuum pillow supporting the hand. 


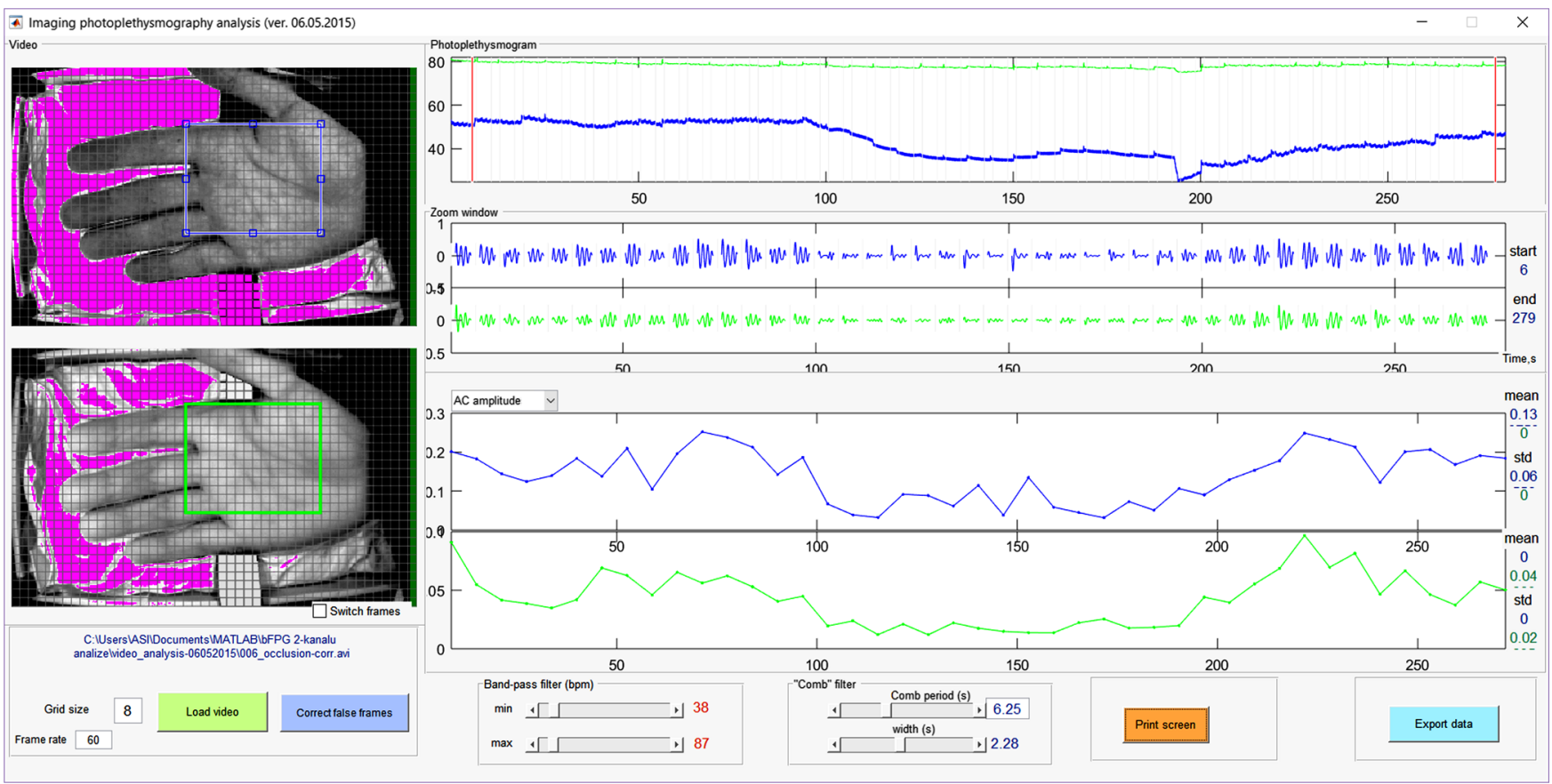

(a)

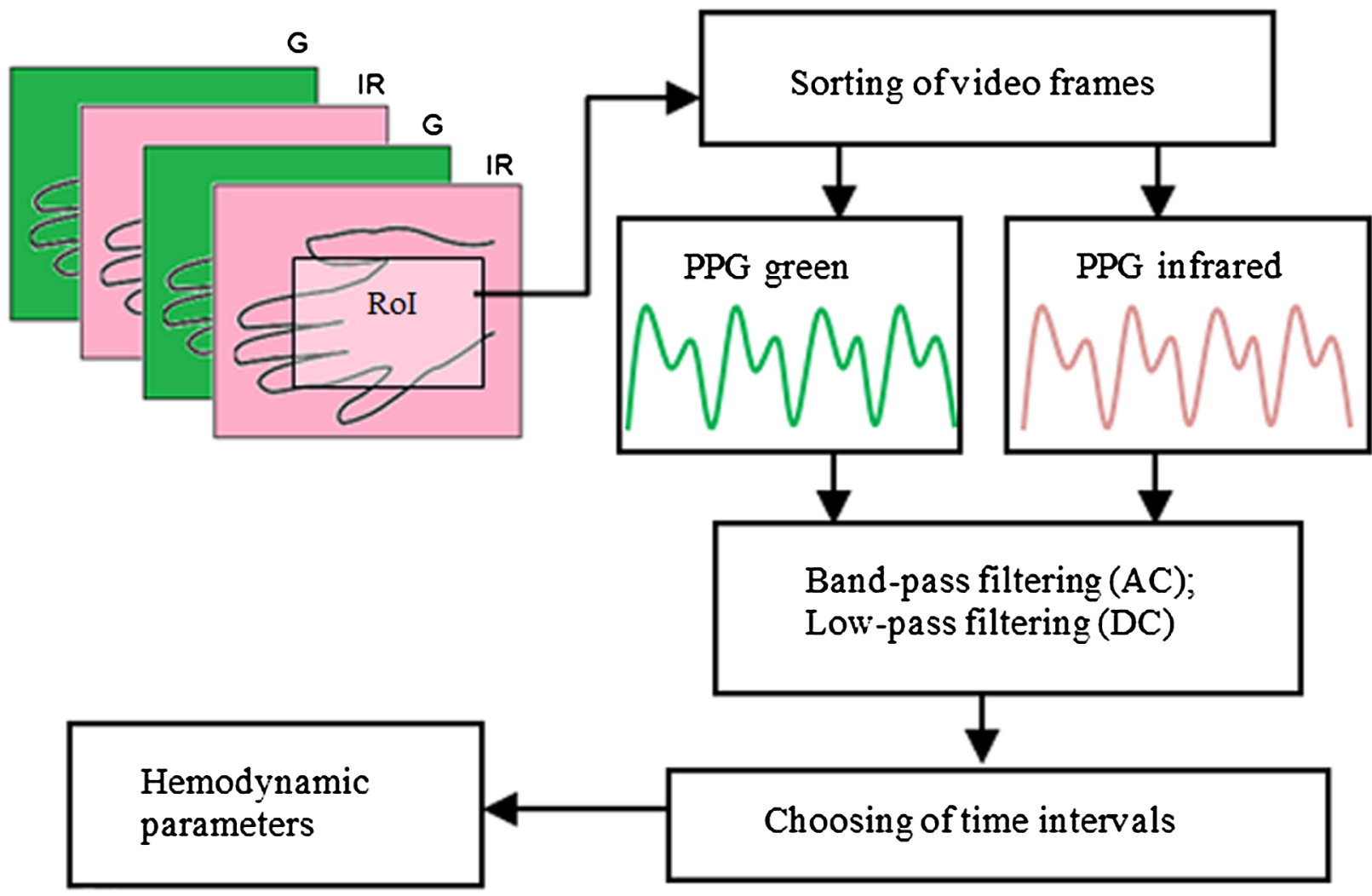

(b)

Fig. 2 (a) MATLAB-based image processing custom developed software and (b) the algorithm for calculation of hemodynamic parameters. The video frames captured at green $(530 \mathrm{~nm})$ and near infrared $(810 \mathrm{~nm})$ light are processed. 
in every heartbeat cycle. The sequence of hemodynamic parameter calculation can be described as follows:

1. The zero-crossing points were obtained from the PPG AC signal; thus, the pulse period of each beat was obtained by following formula:

Pulse period $=\frac{2}{f r}\left(x_{n}-x_{n-1}\right)$,

where $x_{n}$ is a sample number in current beat $n$ and $f r$ is a frame rate of the video signal.

2. The peak-amplitude $A_{n}$ was calculated as the maximum PPG AC signal value in $n$ 'th beat.

3. The mean amplitude was calculated as a standard deviation of PPG signal within the small time interval (10 s):

$$
\text { Amplitude }=\sqrt{\frac{1}{N} \sum_{i=1}^{N} p_{i}^{2}}
$$

where $N$ is the number of samples in time interval and $p_{i}$ is a PPG AC signal in the $i$ 'th sample.

4. Pulse index was calculated as a ratio between PPG AC amplitude and PPG DC signal:

Pulse index $=100 * \frac{\text { Amplitude }}{\frac{1}{N} \sum_{i=1}^{N} D C_{i}}$,

where $D C_{i}$ is a value of PPG DC component in the $i$ 'th sample. ${ }^{29}$

\subsection{Statistical Analysis}

The perfusion characterizing indices from different modalities (iPPG and LDI) are expressed as percentage changes from the baseline. A comparison of perfusion data from three signals was accomplished using analysis of variance, while two signal data ( $\mathrm{PPPG}_{530 \mathrm{~nm}}$ and $\mathrm{iPPG}_{810 \mathrm{~nm}}$ ) were tested with a $t$-test following normality and equal variance analysis. The relationship between different modality time courses was assessed using the Pearson correlation. The significance level was set at $p<0.05$. The data are expressed as means \pm standard error of the mean, unless otherwise stated. The statistical analysis of the data was performed using SigmaPlot 12.5 software (Systat Software Inc.).

\subsection{In Vivo Validation of Imaging Photoplethysmography System}

The in vivo validation study has been approved by the University of Latvia Research Ethics Committee and is in accordance with the guidelines of the Declaration of Helsinki. After being explained all the experimental procedures and the possible risks associated with participation in the study, the subjects gave their written informed consent. The selected subjects were nonsmokers and previously asked to restrain from consuming caffeine or alcohol-containing beverages. In order to assess in vivo capability of imaging PPG, four provocation protocols were accomplished. The tests focused on the predominant effect on the skin superficial subpapillary plexus: local skin heating and topical application of liniment, and with generalized effect on both superficial subpapillary plexus and a deeper plexus: postocclusive reactive hyperemia (PORH) and local anesthesia. All protocols were performed under constant conditions (room temperature $23 \pm 2^{\circ} \mathrm{C}$, relative humidity $50 \pm 5 \%$ ) to ensure adequate microcirculation. Before every test, the subject was acclimatized for $10 \mathrm{~min}$ to the room, comfortably sitting, and the skin surface temperature was measured by infrared thermometer (Raynger MX, Messtechnik, Berlin, Germany).

\subsubsection{Topical application of liniment}

In order to evaluate the ability of iPPG system to discriminate between cutaneous superficial and deep plexus perfusion, hyperemia of superficial plexus was produced with vasodilatory liniment (Transvasin, Seton, United Kingdom) containing nicotinic acid esters-etilnicotinate and hexylnicotinate. Ten healthy, young white Caucasian volunteers (five males, five females, skin type II) were recruited to participate in the protocol. Prior to procedure, the subject was seated on a comfortable reclined chair with the right hand at the dorsal aspect, fixed on the vacuum pillow support (AB, Germa, Sweden) and kept at heart level, with fingers tightly fitted to avoid movements. To mark RoI, the 2-cm-diameter circle was outlined on the dorsal side (dorsum) of the hand (apart from superficial veins) using a surgical marker, so that both the laser Doppler imager (MoorLDI2, Moor Instruments, United Kingdom: $\lambda=785 \mathrm{~nm}$, power $2.5 \mathrm{~mW}$, bandwidth $250 \mathrm{~Hz}$ to $15 \mathrm{kHz}$ ) and iPPG system focused on the same area $\left(1 \mathrm{~cm}^{2}\right)$ at the center. The distances measured for LDI and iPPG were 33 and $20 \mathrm{~cm}$, respectively.

The recording protocol consisted of a 3-min baseline following topical application of liniment (thin smear of Transvasin heat rub cream, Seton, United Kingdom) for $12 \mathrm{~min}$. The same liniment was previously used as an efficient rubefacient in other studies because of its vasoactive ingredients (hexyl nicotinate, ethyl nicotinate). ${ }^{30}$ To avoid optical interference between iPPG and LDI devices during operation, measurements were performed intermittently: $10 \mathrm{~s}$ for LDI scanning (row wise) and $10 \mathrm{~s}$ for iPPG. After processing of the data, individual data points were averaged over $10 \mathrm{~s}$ and consecutive missing values linearly interpolated for both channels, so that the final temporal resolution for peripheral PI was $10 \mathrm{~s}$ (each datapoint).

\subsubsection{Local heating test}

The local heating test has recently gained in popularity as a candidate for routine assessment of endothelial function. ${ }^{6}$ Therefore, it was included in system validation. The study composed of 10 young and healthy volunteers (five males and five females), seated on comfortable reclined chair, with their right hand at the ventral aspect positioned on the vacuum pillow, and kept at heart level. Local heating was induced by adhesive water-filled clear heating probe (VPH3, Moor instruments, United Kingdom) sticking to the palm surface over the "m. abductor pollicis brevis." In doing so, the RoI for both LDI and iPPG systems is set to the same surface in the center of the heating probe. The recording protocol consisted of a 3-min baseline following $12 \mathrm{~min}$ of $40^{\circ} \mathrm{C}$ heating. The interference between optical systems was avoided by performing measurements 
intermittently: $10 \mathrm{~s}$ for LDI scanning (row wise) and $10 \mathrm{~s}$ for iPPG. After processing of data, individual data points were computed, so that the final temporal resolution for PIs was $10 \mathrm{~s}$ (each data point).

\subsubsection{Postocclusive reactive hyperemia test}

To produce considerable alterations of microvascular perfusion and to evaluate the signal for superficial and deep vascular plexuses at "biological zero" conditions, a postocclusive reactive hyperemia test $(\mathrm{PORH})$ test has been utilized by randomly selecting 10 young and healthy volunteers (seven females and three males). The test was performed with the subject comfortably sitting with their right hand slightly extended at palmar aspect and kept at heart level by vacuum pillow support. To prevent any minor movements, fingernails were fixed to support with a double-sided adhesive tape. The occlusion cuff (Hokanson) is placed on the right upper arm. Protocol comprised 2-min resting recording, 3-min suprasystolic arterial occlusion, followed by 5min recovery period. In the analysis software, Rol was selected and peripheral PI was calculated.

\subsubsection{Regional anesthesia test}

In a clinical practice, regional anesthesia (RA) is a routine procedure prior to the surgery. The brachial plexus nerve network block (plexus brachialis peripheral block in axillary level) often is the method of choice for hand surgery; therefore, it was chosen for system validation in clinical settings. Seven patients aged 18 to 40 years undergoing hand surgery received US guided axillary brachial plexus blocks. During the protocol, standard anesthesiology monitoring for RA (ECG, heart rate, blood pressure, pulse oximetry, $\mathrm{CO}_{2}$ level detecting, and temperature control) was performed. During the recording of the procedure, patients were in a supine position, the hand requiring surgery was abducted to $90 \mathrm{deg}$, flexed in elbow level to $90 \mathrm{deg}$

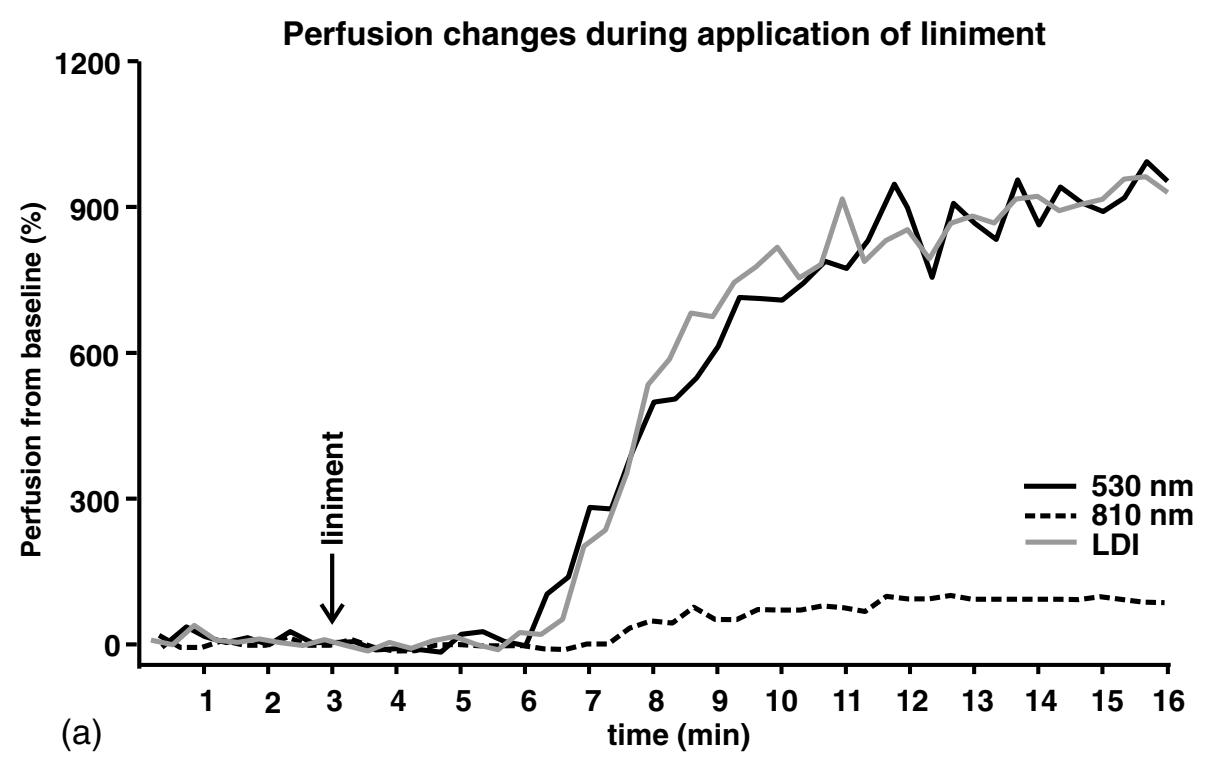

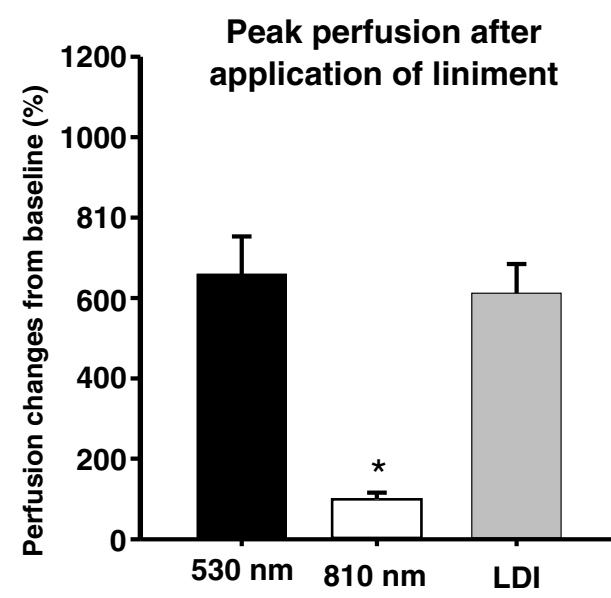

(b)

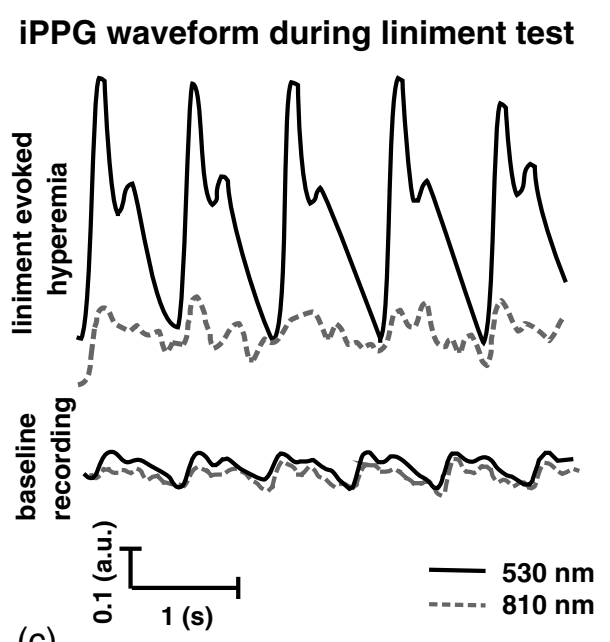

(c)

Fig. 3 (a) Perfusion changes during application of liniment registered with iPPG and LDI, representative example from one subject; arrows indicate the onset of provocation. (b) Peak perfusion changes from the baseline, group mean values \pm standard error of the mean; statistically significant difference at $p<0.05$ are indicated by asterisks. (c) iPPG signal waveforms during baseline recording and liniment evoked hyperemia representative example from one subject. 
and upturned to $30 \mathrm{deg}$. The dorsal aspect of the hand was comfortably adjusted in vacuum pillow and fingernails fixed with a double-sided adhesive tape. Video from the palm surface was recorded $1 \mathrm{~min}$ before, during, and $10 \mathrm{~min}$ after the block. The block was produced by administrating a combination of Sol.Lidocaini 2\%, $(10 \mathrm{ml})$ and Sol.Bupivacaini 0.5\% (10 ml). After the measurement, the hand was removed and the anesthesia level was controlled with "conventional" methods, e.g., ice.

\section{Results}

In total, 38 subjects were evaluated in four different protocols. The mean age of the subject group was $26 \pm 10$ years, body mass index: $24.3 \pm 2.4 \mathrm{~kg} / \mathrm{m}^{2}$; all volunteers had a normal resting heart rate $65.7 \pm 7.4 \mathrm{BPM}$, and arterial blood pressure (systolic: $125 \pm 10 \mathrm{mmHg}$; diastolic: $79 \pm 7 \mathrm{mmHg}$ ). Skin temperature during the measurement slightly varied from protocol to protocol, ranging from $32^{\circ} \mathrm{C}$ to $28^{\circ} \mathrm{C}$. In all protocols, consistent blood perfusion data have been acquired. A detailed description of the results is provided in the following.

\subsection{Topical Application of Liniment}

The topical application of liniment is a decisive test in the present study as it is able to induce selective vasodilation of superficial microvessels. ${ }^{31}$ Within 3 to 4 min after application of the liniment, reddening of skin (flushing) appeared, indicating hyperemia. The response began with the rapid vasodilation 3 to 4 min after application and reached its plateau 6 to 7 min later, as described elsewhere. ${ }^{31,32}$ All subjects individually demonstrated a statistically significant increase of all three signals $\left(\mathrm{iPPG}_{530 \mathrm{~nm}}, \mathrm{iPPG}_{810 \mathrm{~nm}}, \mathrm{LDI}\right)$ from the baseline. The representative illustration of relative cutaneous perfusion changes during the test is depicted in Fig. 3(a). The subject group relative peak perfusion values are shown in Fig. 3(b) and are iPPG $_{530 \mathrm{~nm}}=$ $659 \pm 297 \% ; \mathrm{iPPG}_{810 \mathrm{~nm}}=99 \pm 52 \%$; LDI $=611 \pm 228 \%$.

Although an increase in the $\mathrm{PPG}_{810 \mathrm{~nm}}$ signal was negligible in comparison to PPG $_{530 \mathrm{~nm}}$, as depicted in Fig. 3(a), it has been noticed that the $\mathrm{iPPG}_{810 \mathrm{~nm}}$ waveform during peak perfusion (hyperemia) was noisy at low amplitude in contrast to that of

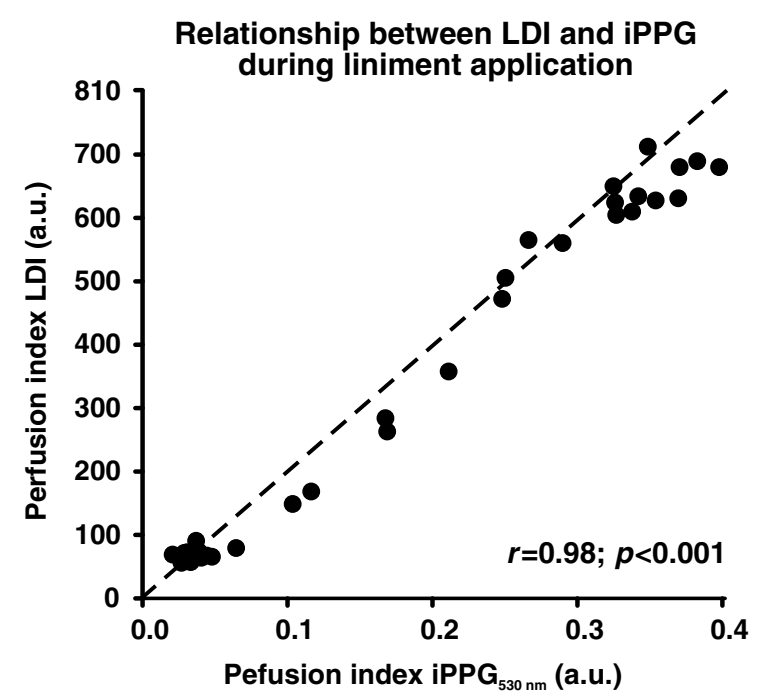

Fig. 4 Relationship between perfusion obtained from LDI and ${ }_{i P P G} G_{530 \mathrm{~nm}}$ during liniment application test, representative example from one subject.
iPPG $_{530 \mathrm{~nm}}$, see Fig. 3(c). The observed similarity between $\mathrm{PPG}_{530 \mathrm{~nm}}$ and LDI perfusion changes has been confirmed by their high correlation (group mean $r=0.98 \pm 0.01, p<0.001$ ), as depicted in Fig. 4.

\subsection{Local Heating Test}

The local heating of nonglabrous skin produced a significant increase of cutaneous perfusion from the baseline in all subjects for $\mathrm{iPPG}_{530 \mathrm{~nm}}, \mathrm{iPPG}_{810 \mathrm{~nm}}$, and LDI $\sim 6 \mathrm{~min}(6.0 \pm 1.5 \mathrm{~min})$ following the onset of warming. The largest increase of perfusion was observed for $\mathrm{iPPG}_{530 \mathrm{~nm}}$, and LDI signals, with the same trend forming initial peak with the subsequent nadir, as depicted in Fig. 5(a) (13 to 16 min). A similar response was noted for the $\mathrm{iPPG}_{530 \mathrm{~nm}}$ waveform-signal amplitude as it increased during the heating-evoked hyperemia [see Fig. 5(c)]. The mean relative peak perfusion is depicted in Fig. 5(b) and values were $\mathrm{iPPG}_{530 \mathrm{~nm}}=370 \pm 136 \%$; $\mathrm{iPPG}_{810 \mathrm{~nm}}=$ $123 \pm 29 \%$; LDI $=530 \pm 327 \%$. There was no statistically significant difference between $\mathrm{iPPG}_{530 \mathrm{~nm}}$ and LDI.

Correlation analyses revealed a close relationship $(r=0.93 \pm 0.01 ; p<0.001)$ between LDI and $\mathrm{iPPG}_{530 \mathrm{~nm}}$ PI. The representative data for one subject are displayed in Fig. 6.

\subsection{Postocclusive Reactive Hyperemia Test}

The occlusion test has been selected for the present study to evoke an increase of perfusion in both superficial and deep circulation of glabrous skin. Subjects showed a typical perfusion trend for both iPPG signals, comprising relatively flat baseline, rapid decline at the onset of occlusion, and PORH peak (reactive hyperemia), as shown in Fig. 7(a). The iPPG waveform amplitudes differed in all three conditions, as depicted in Fig. 7(c).

There was no significant difference between perfusion obtained from iPPG $530 \mathrm{~nm}$ and $\mathrm{iPPG}_{810 \mathrm{~nm}}$; hence, for volunteers with higher sympathetic tone and subsequently lower baseline, the so-called "biological zero" during occlusion period was higher, exhibiting minor difference between iPPG signals. The group mean values for the relative PORH peak did not significantly differ with the slightly higher $\mathrm{PPG}_{530 \mathrm{~nm}}=94 \pm$ $24 \%$ and lower $\mathrm{PPPG}_{810 \mathrm{~nm}}=71 \pm 23 \%$, see Fig. $7(\mathrm{~b})$.

\subsection{Regional Anesthesia Test}

The recording of glabrous skin perfusion during rest and brachial plexus block is important as an appraisal for the potential clinical application of our iPPG system. The expected effect is a decrease in sympathetic vascular tone resulting in vasodilation and subsequent elevation of perfusion in superficial and deep microvasculature.

The gradual increase in perfusion in both iPPG channels was observed 5 to 7 min following successfully administrated local anesthetics, where the plateau phase was reached in $\sim 15$ to $16 \mathrm{~min}$ after administration [see Fig. 8(a)]. The perfusion response timescale slightly differs across the patients, varying depending on heterogeneity of patient group and variance of administration procedure. The patient group mean relative peak perfusion did not differ $\left(\mathrm{iPPG}_{530 \mathrm{~nm}}=413 \pm 208 \%\right.$; $\mathrm{iPPG}_{810 \mathrm{~nm}}=359 \pm 191 \%$ ), indicating similar responses in superficial and deeper vasculature situated in dermal and hypodermal layers [Fig. 8(b)]. The same tendency is seen in iPPG waveform, as depicted in Fig. 8(c). 

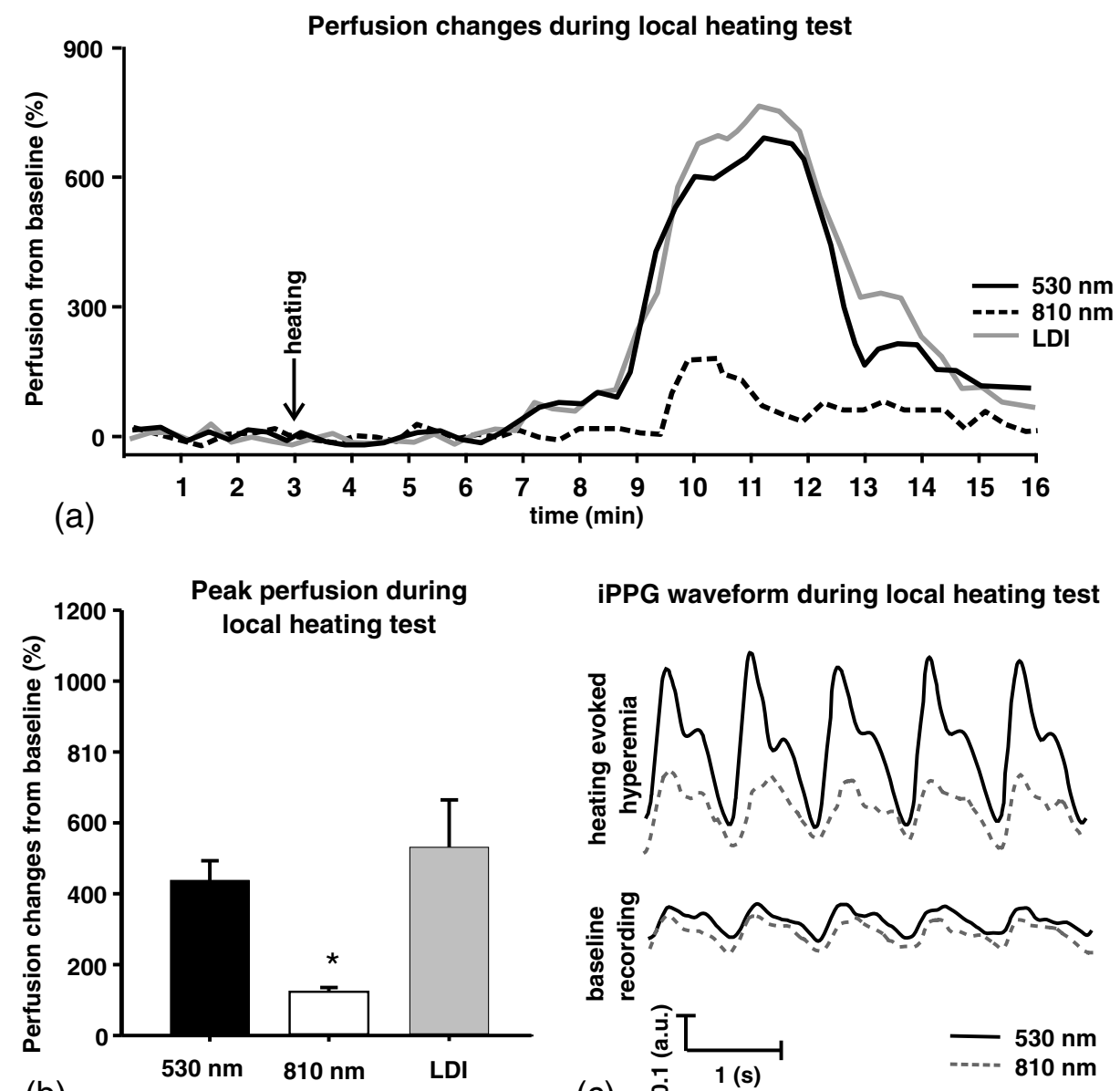

iPPG waveform during local heating test

(b)

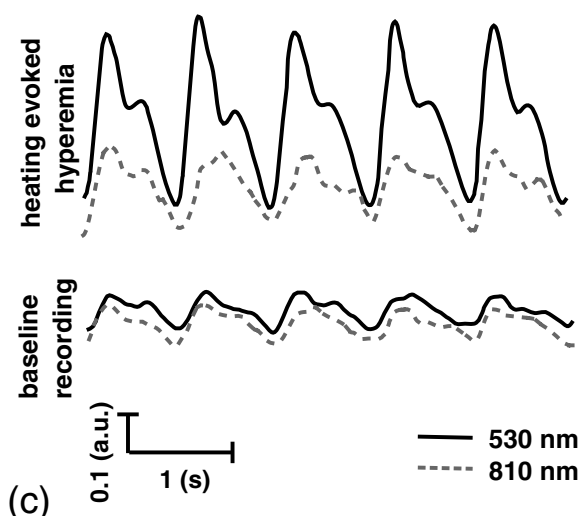

Fig. 5 (a) Perfusion changes during local heating test registered with iPPG and LDI, representative example from one subject, arrows indicate the onset of provocation. (b) Peak perfusion changes from the baseline, group mean values \pm standard error of the mean, statistically significant difference at $p<0.05$ is indicated by asterisks. (c) iPPG signal waveforms during baseline recording and local heating induced hyperemia, representative date from one subject.

\section{Relationship between LDI and IPPG} during local heating test

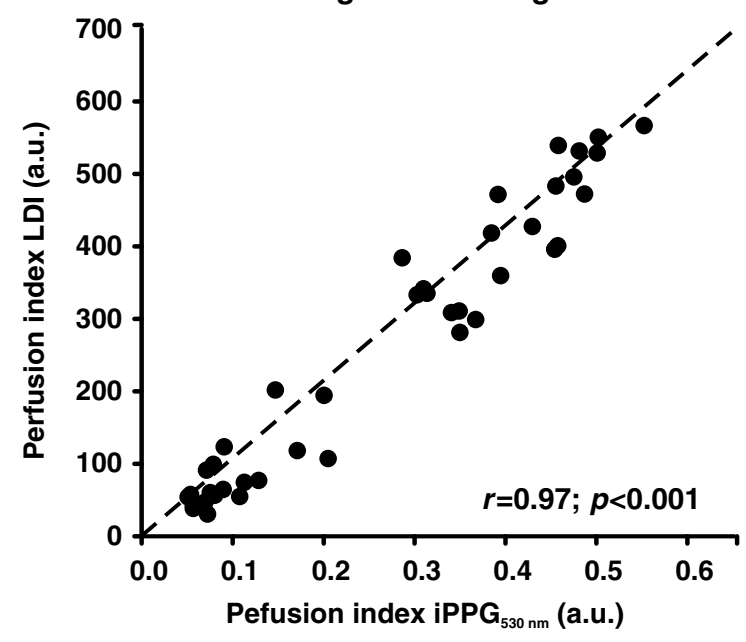

Fig. 6 Relationship between perfusion registered with LDI and iPPG ${ }_{530} \mathrm{~nm}$, representative example from one subject.

\section{Discussion}

This study reveals the feasibility of the iPPG system in registering blood perfusion from two functionally and morphologically different layers of cutaneous microvasculature-a superficial subpapillary plexus and deeper plexus at the dermis-hypodermis junction. In our iPPG system, the theoretically expected optical penetration depth (radiation attenuated to $1 / \mathrm{e}$ of the incident radiation intensity) for $\mathrm{iPPG}_{530 \mathrm{~nm}}$ was $0.9 \mathrm{~mm}$ and for

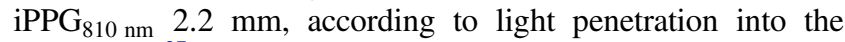
skin model. ${ }^{27}$ Considering the thicknesses of different cutaneous layers in glabrous and nonglabrous skin and distribution of blood vessels recently obtained by high-resolution ultrasound $^{33}$ and ultrahigh sensitive optical microangiography technique ${ }^{34}$ the upper dermal circulatory plexus is situated $\sim 0.40$ to $0.45 \mathrm{~mm}$ below the skin surface, which is within the recording range of $\mathrm{PPPG}_{530 \mathrm{~nm}}$, whereas the subcutaneous arteriovenous plexus is located $\sim 0.78$ to $1.10 \mathrm{~mm}$ and therefore can be reached by $\mathrm{iPPG}_{810 \mathrm{~nm}}$. However, in the present experimental design, identification of the vascular plexus is based on specific physiological responses induced by known provocation tests rather than theoretical modeling and calculation. Accordingly, for a selective increase of blood perfusion in the superficial plexus, 


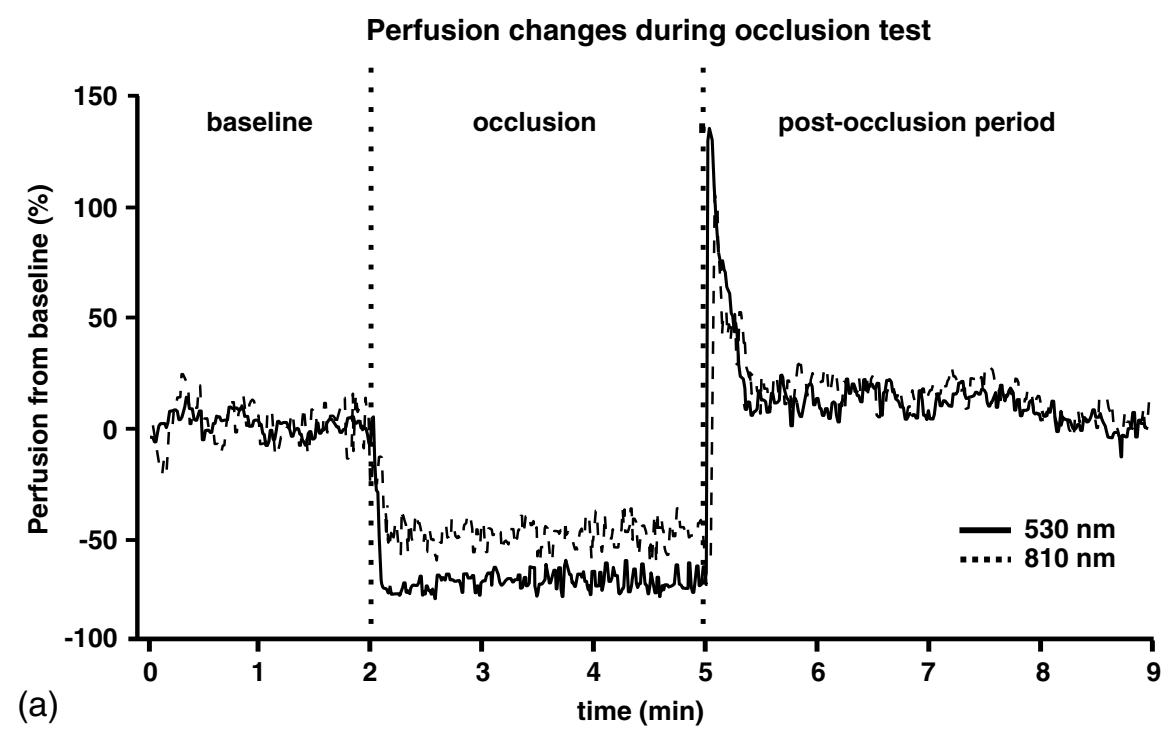

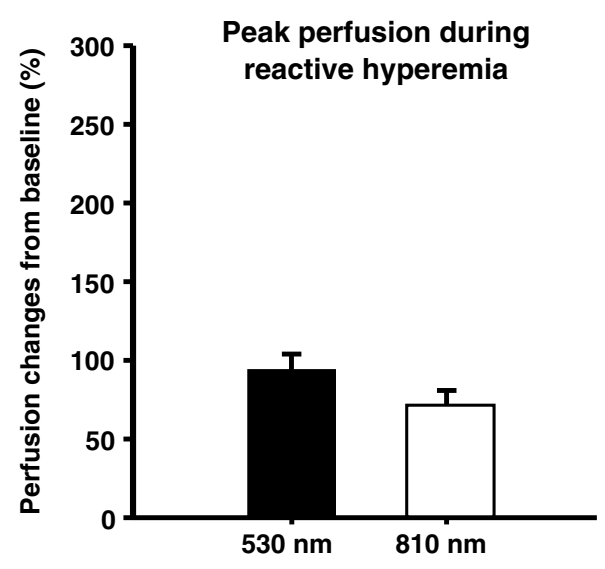

(b)
IPPG waveform during occlusion test

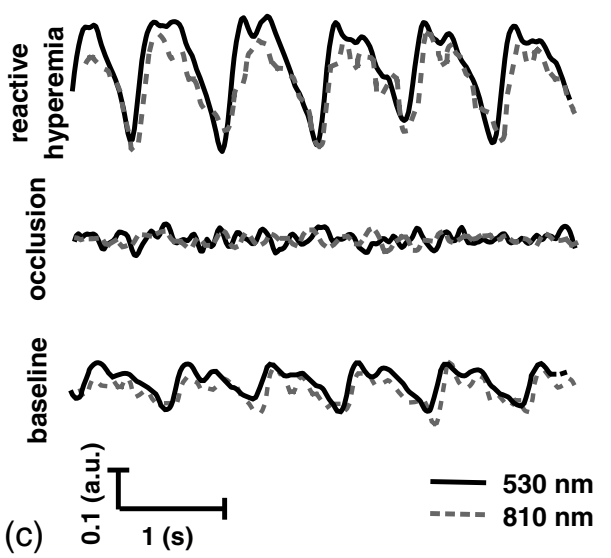

Fig. 7 (a) Perfusion changes during occlusion test registered with iPPG and LDI, representative example from one subject; dotted lines indicate various stages (baseline, occlusion, postocclusion). (b) Peak perfusion changes from the baseline during reactive hyperemia test, group mean values \pm standard error of the mean; statistically significant difference at $p<0.05$ is indicated by asterisks. (c) iPPG signal waveforms during baseline, arterial occlusion and postocclusion period.

topical application of vasodilatory liniment and local skin heating tests were utilized, while generalized vasodilation in both plexuses was induced by POHR and local anesthesia (block or sympathetic efferentation). To the best of our knowledge, the present study provides the most extensive evaluation of bispectral (530- and 810-nm light sources) iPPG in in vivo conditions during different protocols. The detailed discussion on the protocols is provided below.

\subsection{Topical Application of Liniment}

The skin flushing phenomenon (reddening) observed in the present study after topical application of vasodilatory liniment is characterized by a dramatic increase in perfusion and number of functional capillaries in superficial subpapillary plexus within the upper dermis, where absorption of hemoglobin at $530 \mathrm{~nm}$ is high due to the very dense vascular network. During resting conditions and comfortable temperatures, this plexus maintains relatively low in perfusion as it serves mostly for heat transfer purposes. When the liniment is applied on the skin, the active ingredients, nicotinic acid esters (ethyl nicotinate, hexyl nicotinate), are converted to niacin (nicotinic acid) by cutaneous esterases after penetration into the skin. ${ }^{35}$ At least two different physiological mechanisms account for the vascular component of the flushing, one involving activation of niacin receptors (GPR109A) in dermal Langerhans cells and keratinocytes, ${ }^{36-39}$ leading to secretion of prostaglandins, the other activates the Capsaicin receptor (TRPV1) pathway. ${ }^{40,41}$ In the end, both pathways produce vasodilation of arterioles and precapillary sphincters and subsequently increased capillary perfusion. The protocols utilizing topical application of rubefacients, such as nicotinic acid, have been used for decades in pharmacological, dermatological, and biophotonic studies. ${ }^{28,30-32,42,43}$ Hence, contactless cutaneous perfusion measurement at different depth is still the exclusive option due to the lack of appropriate instrumentation, and only few studies are available employing two-bandwidth LDI. For example, Jacobi et al. ${ }^{13,35}$ utilized a two-bandwidth laser Doppler ( 0.5 to $5 \mathrm{kHz}$ and 5 to $30 \mathrm{kHz}$ ) instrument for measurement of cutaneous microcirculation at two different depths. ${ }^{13,35}$ Much like our results, the authors 


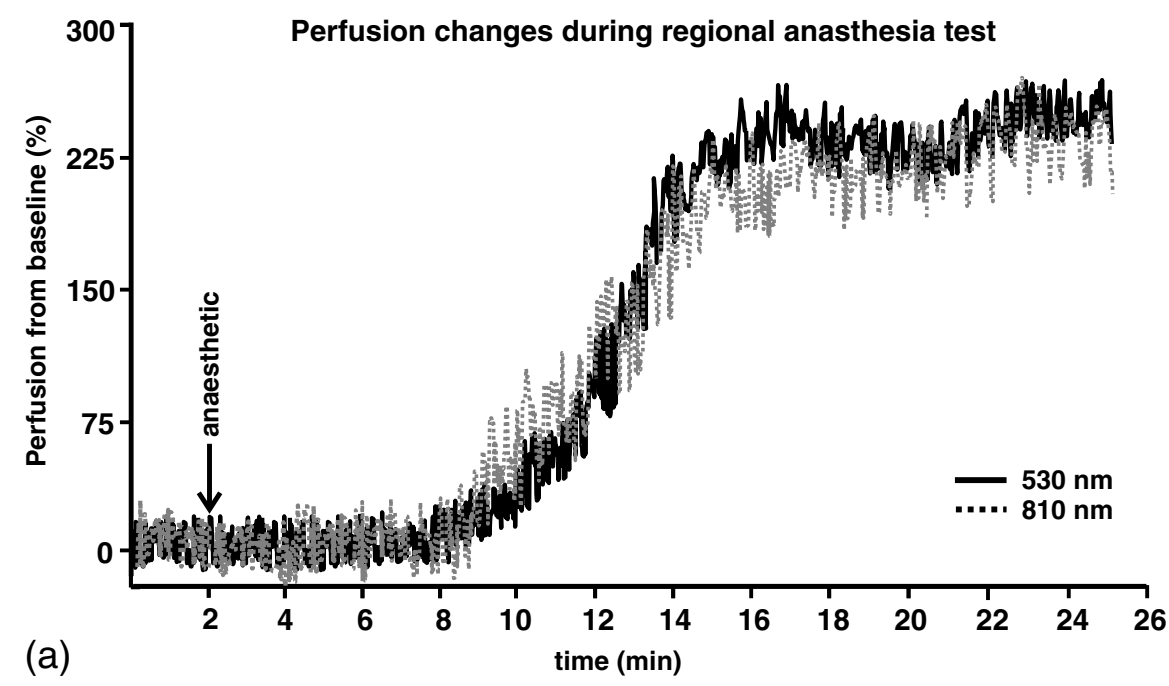

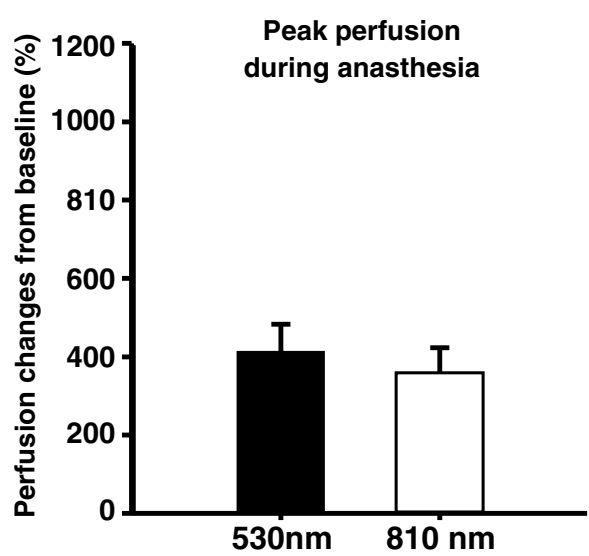

(b)

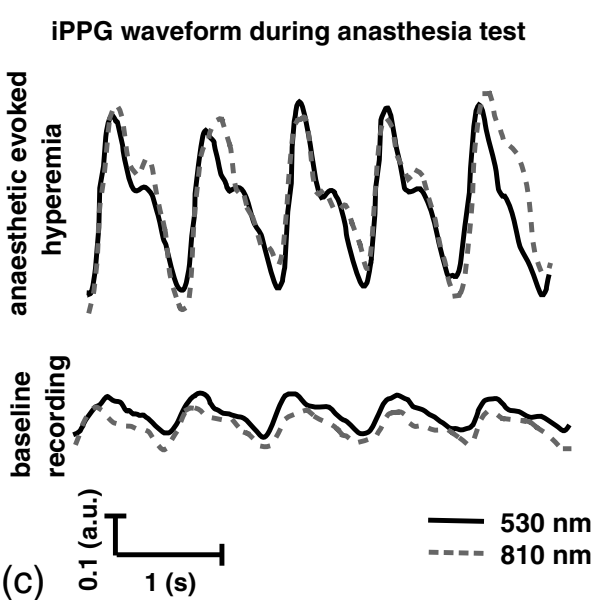

Fig. 8 (a) Perfusion changes during RA test obtained from iPPG and LDI, representative example from one subject, arrow indicates the onset of provocation. (b) Peak perfusion changes from the baseline, group mean values \pm standard error of the mean; statistically significant difference at $p<0.05$ is indicated by asterisks. (c) iPPG signal waveforms during baseline recording and anesthetic evoked hyperemia.

reported higher perfusion in superficial capillaries compared to the deeper vasculature following liniment application. A highamplitude perfusion signal in the $\mathrm{iPPG}_{530 \mathrm{~nm}}$ channel during liniment test is produced by substantial vasodilation in the relatively dense capillary network. The same perfusion trend was observed in our data from a different modality, LDI signal [Fig. 3(a)], with the relatively wide measurement range (signal acquisition ranges from 0 to $1.5 \mathrm{~mm}$ ), and hence, a different operating principle.

Conversely, the weak $\mathrm{iPPG}_{810 \mathrm{~nm}}$ signal during the liniment test indicates minor involvement of deeper cutaneous vessels in the flushing response, reported by Jacobi et al. The results obtained in the liniment test indicate the capacity of our iPPG system to register superficial subpapillary blood perfusion utilizing the 530-nm light source.

\subsection{Local Heating Test}

The response of skin blood flow to local heating includes complex interactions between neural components and locally produced chemical messengers. ${ }^{44}$ The typical response to local heating of nonglabrous skin is an initial peak (following $10 \mathrm{~min}$ of heating), a subsequent drop to nadir, and a further vasodilation to a sustained plateau (after $20 \mathrm{~min}$ ). ${ }^{45}$ In our protocol, after a relatively short heating time, only the first peak and part of the nadir were observed, while lacking a sustained plateau. The possible cause is the different vasoreactivity of glabrous and nonglabrous skin, which is mentioned in the study of Metzler-Wilson et al. ${ }^{46}$ The similarities between the LDI and $\mathrm{PPG}_{530 \mathrm{~nm}}$ traces provide evidence that subjects exhibited consistent vascular response, predominantly in superficial vascular plexus situated close to dermal epidermal junction, where densities of capillaries are high. While the blunted response of $\mathrm{iPPG}_{810 \mathrm{~nm}}$ reflect the perfusion of dermal-hypodermal junction, comprising less dense, relatively large caliber vessels. Thee perfusion changes occurring closer to the skin surface will be masked at longer wavelengths by the perfusion changes at deeper levels. This may partly explain the wavelength dependence of the iPPG signal. To the best of our knowledge, the simultaneous blood perfusion registering in superficial and deep plexuses has never been demonstrated by means of the iPPG technique, which makes it difficult to compare our findings. 
However, the feasibility of single spectral (green light) iPPG during thelocal heating test has been previously reported in our earlier study. ${ }^{47}$ The results of th local heating protocol largely support the findings obtained in the liniment protocol, similarly indicating the feasibility of the iPPG system to selectively acquire perfusion from the superficial vascular plexus utilizing a 530-nm light source.

\subsection{Postocclusive Reactive Hyperemia Test}

The occlusion test comprising PORH is often used as a provocation test due to its convenience and strong regional vascular response. ${ }^{48}$ The postocclusive hyperemia phenomenon occurs after the restoration of blood supply due to marked dilatation, in some circumstances even reaching maximal vasodilatory capacity of the vascular bed (usually following $15 \mathrm{~min}$ of occlusion). Two major physiological mechanisms are equally contributing to PORH: direct relaxation of vascular smooth muscle by endothelial hyperpolarizing factor and reflectory vasodilation due to stimulation of sensory nerve endings by accumulated metabolites. ${ }^{49}$ In the present protocol, the occlusion time was much shorter ( $3 \mathrm{~min}$ instead of $15 \mathrm{~min}$ ) to minimize subject discomfort and solely reveal differences between perfusion in deep and superficial vascular plexuses. Previous studies confirmed the strong vasodilation of the entire occluded vascular bed, comprising small arteries and arterioles, supplying skeletal muscle and skin. ${ }^{30,50,51}$ This explains the observed negligible difference in relative peak perfusion for $\mathrm{iPPG}_{530 \mathrm{~nm}}$ and $\mathrm{iPPG}_{810 \mathrm{~nm}}$, as superficial dermal and deep cutaneous vessels almost equally respond during PORH. Nevertheless, the slightly larger perfusion for superficial microvasculature during PORH may be attributed partly to different vasoreactivity in dermal end subcutaneous microvascular plexuses, partly due to higher absorption of dense superficial capillaries. Unfortunately, the results were inconclusive, as no studies were identified regarding responses in superficial and deep cutaneous perfusion registered by means of the iPPG technique. However, in studies utilizing single spectral iPPG, the same trend of perfusion changes was shown during baseline, occlusion, and PORH. ${ }^{51}$ Summarizing the present findings, we consistently demonstrate that the occlusion test, as expected, induced detectable generalized responses of perfusion in both superficial and deep vascular layers in the glabrous skin.

\subsection{Regional Anesthesia Test}

The local anesthesia (plexus brachialis block) protocol was included in evaluation of the iPPG system for two reasonsto assess the sensitivity of the iPPG system during generalized skin sympathetic blockage and to asses our system in real clinical conditions. Despite the fact that the brachial plexus blockade for upper-extremity surgery is a very common clinical procedure, ${ }^{52}$ there are quite a few papers describing iPPG for anesthesia monitoring. ${ }^{53,54}$ Our findings are consistent with results obtained in several studies utilizing LDI $^{55,56}$ and a thermal camera,${ }^{57}$ indicating increased perfusion during the brachial plexus block in both superficial dermal ( PPG $_{530 \mathrm{~nm}}$ ) and deeper vascular layers $\left(\mathrm{iPPG}_{810 \mathrm{~nm}}\right)$. The data are consistent with early findings, which state that a block of sympathetic nerves is associated with a sudden drop of peripheral sympathetic (vasoconstrictor) nerve activity, ${ }^{58}$ thereby leading to vasodilation, increased blood flow, and increase in tissue/skin temperature. ${ }^{56,57}$ The results obtained in this protocol are similar to that of PORH because of the generalized response of superficial and deep cutaneous vascular plexuses, confirming that our designed iPPG system could be used in clinical settings.

\section{Conclusions}

Our results indicate the high potential of the bispectral iPPG system in assessment of cutaneous blood perfusion from functionally and morphologically different microvascular plexuses and superficial subpapillary plexus, comprising dense capillary loops and a deeper plexus at the dermis-hypodermis junction, containing arteries and arterioles. This system also can be utilized in clinical settings as a cost-effective alternative to the LDI in assessing microcirculatory perfusion and particularly in the cases where registering of superficial and deep cutaneous perfusion is crucially important.

\section{Limitations of the Study}

Despite careful planning of the experimental design and accurate implementation of all procedures, there are limitations that may potentially interfere with the present findings; therefore, these results should be interpreted with caution. One limitation of this study is validation of iPPG system utilizing living tissue (in vivo), as the individual subjects may have slightly different thicknesses of skin layers and different optical properties. However, we believe that the differences in the present study were negligible ${ }^{59}$ because the individually observed effects were consistent and did not significantly differ from the group mean value. Moreover, the use of more precise and predictable model-tissue phantoms in our study could be rather arguable, as they are not able to mimic real physiological microcirculatory responses because of its high complexity.

Another limitation of this study is the inability to perform a provocation procedure, where the deep cutaneous perfusion changes while the superficial remain the same. There are no acceptable standard physiological tests available that would selectively increase cutaneous perfusion in the deeper plexus, without influencing superficial subpapillary microcirculation. ${ }^{4}$ In the present study, the ability of the custom-designed iPPG system to determine perfusion in different cutaneous microcirculatory plexuses, superficial subpapillary and deep dermal, has been demonstrated by means of well-known physiological provocations: selectively increasing superficial perfusion (local heating and topical application of rubefacient) and simultaneously increasing perfusion in both superficial and deep microvasculature (arterial occlusion test).

\section{Acknowledgments}

Financial support (Grant No. 2014/0036/2DP/2.1.1.1.0/14/ APIA/VIAA/020) from the European Regional Development Fund is highly appreciated.

\section{References}

1. M. Roustit and J. L. Cracowski, "Assessment of endothelial and neurovascular function in human skin microcirculation," Trends Pharmacol. Sci. 34(7), 373-384 (2013).

2. D. Roosterman et al., "Neuronal control of skin function: the skin as a neuroimmunoendocrine organ," Physiol. Rev. 86(4), 1309-1379 (2006).

3. C. Bergek, J. H. Zdolsek, and R. G. Hahn, "Non-invasive blood haemoglobin and plethysmographic variability index during brachial plexus block," Br. J. Anaesth. 114(5), 812-817 (2015).

4. H. Lenasi, "Assessment of human skin microcirculation and its endothelial function using laser doppler flowmetry," Medical Imaging, F. Okechukwu Erondu, Ed., p. 412, InTech (2011). 
5. J. L. Saumet et al., "Cutaneous laser-Doppler flowmetry: influence of underlying muscle blood flow," J. Appl. Physiol. 65(1), 478-481 (1988).

6. G. Mahé et al., "Assessment of skin microvascular function and dysfunction with laser speckle contrast imaging," Circ. Cardiovasc. Imaging 5(1), 155-163 (2012).

7. D. Briers et al., "Laser speckle contrast imaging: theoretical and practical limitations," J. Biomed. Opt. 18(6), 066018 (2013).

8. J. O'Doherty et al., "Sub-epidermal imaging using polarized light spectroscopy for assessment of skin microcirculation," Skin Res. Technol. 13(4), 472-484 (2007).

9. J. Wang et al., "Assessment of optical clearing induced improvement of laser speckle contrast imaging," J. Innov. Opt. Health Sci. 3(3), 159-167 (2010).

10. O. B. Thompson and M. K. Andrews, "Tissue perfusion measurements: multiple-exposure laser speckle analysis generates laser Doppler-like spectra," J. Biomed. Opt. 15(2), 027015 (2010).

11. R. Liu, J. Qin, and R. K. Wang, "Motion-contrast laser speckle imaging of microcirculation within tissue beds in vivo," J. Biomed. Opt. 18(6), 060508 (2013).

12. J. Wang et al., "Review: tissue optical clearing window for blood flow monitoring," IEEE J. Sel. Top. Quantum Electron. 20(2), 92-103 (2014).

13. U. Jacobi et al., "Comparison of blood flow to the cutaneous temperature and redness after topical application of benzyl nicotinate," J. Biomed. Opt. 11(1), 014025 (2006).

14. K. M. Asplund et al., "Photon path depth in tissue phantoms: a comparison of visible and near-infrared (NIR) wavelengths," Proc. SPIE 8945, 89450D (2014).

15. V. V. Tuchin et al., "Finger tissue model and blood perfused skin tissue phantom," Proc. SPIE 7898, 78980Z (2011).

16. C. Chen et al., "Recovering the superficial microvascular pattern via diffuse reflection imaging: phantom validation," Biomed. Eng. Online 14(1), 1-14 (2015).

17. M. S. Wróbel et al., "Multi-layered tissue head phantoms for noninvasive optical diagnostics," J. Innov. Opt. Health Sci. 8(3), 1541005 (2015).

18. A. V Bykov et al., "Skin phantoms with realistic vessel structure for OCT measurements," Proc. SPIE 7376, 73760F (2010).

19. M. Kumar, A. Veeraraghavan, and A. Sabharwal, "DistancePPG: robust non-contact vital signs monitoring using a camera," Biomed. Opt. Express 6(5), 1565 (2015).

20. K. Humphreys, T. Ward, and C. Markham, "A CMOS camera-based pulse oximetry imaging system," in 2005 IEEE Engineering in Medicine and Biology 27th Annual Conf., Vol. 4, pp. 3494-3497, IEEE (2005).

21. K. Humphreys, C. Markham, and T. E. Ward, "A CMOS camera-based system for clinical photoplethysmographic applications," Proc. SPIE 5823, 88-95 (2005).

22. Y. Sun et al., "Use of ambient light in remote photoplethysmographic systems: comparison between a high-performance camera and a lowcost webcam," J. Biomed. Opt. 17(3), 037005 (2012).

23. H.-Y. Wu et al., "Eulerian video magnification for revealing subtle changes in the world," ACM Trans. Graph. 31(4), 1-8 (2012).

24. X. He, R. A. Goubran, and X. P. Liu, "Using Eulerian video magnification framework to measure pulse transit time," in IEEE Int. Symp. on Medical Measurements and Applications, pp. 50-53 (2014).

25. S. Hu et al., "Feasibility of imaging photoplethysmography," in 2008 Int. Conf. on BioMedical Engineering and Informatics, Vol. 2, pp. $72-75$ (2008).

26. A. A. Kamshilin et al., "A new look at the essence of the imaging photoplethysmography," Sci. Rep. 5, 10494 (2015).

27. A. N. Bashkatov et al., "Optical properties of human skin, subcutaneous and mucous tissues in the wavelength range from 400 to $2000 \mathrm{~nm}$," J. Phys. D. Appl. Phys. 38(15), 2543-2555 (2005).

28. M. L. Sandberg, M. K. Sandberg, and J. Dahl, "Blood flow changes in the trapezius muscle and overlying skin following transcutaneous electrical nerve stimulation," Phys. Ther. 87(8), 1047-1055 (2007).

29. A. Pinto Lima, P. Beelen, and J. Bakker, "Use of a peripheral perfusion index derived from the pulse oximetry signal as a noninvasive indicator of perfusion," Crit. Care Med. 30(6), 1210-1213 (2002).
30. M. Sandberg et al., "Non-invasive monitoring of muscle blood perfusion by photoplethysmography: evaluation of a new application," Acta Physiol. Scand. 183(4), 335-343 (2005).

31. G. P. Fulton, E. M. Farber, and A. P. Moreci, "The mechanism of action of rubefacients," J. Invest. Dermatol. 33(6), 317-325 (1959).

32. A. Caselli et al., "Topical methyl nicotinate-induced skin vasodilation in diabetic neuropathy," J. Diabetes Complications 17(4), 205-210 (2003).

33. K. S. Frahm et al., "Spatial temperature distribution in human hairy and glabrous skin after infrared $\mathrm{CO}_{2}$ laser radiation," Biomed. Eng. Online 9(1), 69 (2010).

34. L. An, J. Qin, and R. K. Wang, "Ultrahigh sensitive optical microangiography for in vivo imaging of microcirculations within human skin tissue beds," Opt. Express 18(8), 8220-8228 (2010).

35. U. Jacobi et al., "Kinetics of blood flow after topical application of benzyl nicotinate on different anatomic sites," Arch. Dermatol. Res. 298(6), 291-300 (2006).

36. V. S. Kamanna, S. H. Ganji, and M. L. Kashyap, "The mechanism and mitigation of niacin-induced flushing," Int. J. Clin. Pract. 63(9), 13691377 (2009).

37. R. H. Guy et al., "Noninvasive assessments of the percutaneous absorption of methyl nicotinate in humans," J. Pharm. Sci. 72(9), 1077-1079 (1983).

38. H. Silva et al., "Exploring human in vivo microcirculation with methyl nicotinate in different perfusion conditions," Biomed Biopharm Res 11(2), 207-214 (2014).

39. C. D. Meyers et al., "Nicotinic acid induces secretion of prostaglandin D2 in human macrophages: an in vitro model of the niacin flush," Atherosclerosis 192(2), 253-258 (2007).

40. L. Ma et al., "Nicotinic acid is a common regulator of heat-sensing TRPV1-4 ion channels," Sci. Rep. 5, 1-8 (2015).

41. L. Ma et al., "Nicotinic acid activates the capsaicin receptor TRPV1: potential mechanism for cutaneous flushing," Arterioscler. Thromb. Vasc. Biol. 34(6), 1272-1280 (2014).

42. J. Hagblad et al., "Technical issues related to the long-term monitoring of blood flow at different depths using LDF and PPG," Physiol. Meas. 33(6), 985-996 (2012).

43. S. Bergstrand et al., "Blood flow measurements at different depths using photoplethysmography and laser Doppler techniques," Ski. Res. Technol. 15(2), 139-147 (2009).

44. J. M. Johnson, C. T. Minson, and D. L. Kellogg, "Cutaneous vasodilator and vasoconstrictor mechanisms in temperature regulation," Compr. Physiol. 4(1), 33-89 (2014).

45. C. T. Minson, L. T. Berry, and M. J. Joyner, "Nitric oxide and neurally mediated regulation of skin blood flow during local heating," J. Appl Physiol 91(4), 1619-1626 (2001).

46. K. Metzler-Wilson et al., "Differential vasodilatory responses to local heating in facial, glabrous and hairy skin," Clin. Physiol. Funct. Imaging 32(5), 361-366 (2012).

47. U. Rubins et al., "Real-time photoplethysmography imaging system," in 15th NBC on Biomedical Engineering \& Medical Physics, Vol. 34, K. Dremstrup, S. Rees, and M. Ø. Jensen, Eds., pp. 183-186, Springer, Berlin (2011).

48. D. Jakovels, U. Rubins, and J. Spigulis, "LASCA and PPG imaging for non-contact assessment of skin blood supply," Proc. SPIE 8668, 866849 (2013).

49. F. Iredahl et al., "Non-invasive measurement of skin microvascular response during pharmacological and physiological provocations," PLoS One 10(8), e0133760 (2015).

50. S. Farnebo et al., "Hyperaemic changes in forearm skin perfusion and RBC concentration after increasing occlusion times," Microvasc. Res. 80(3), 412-416 (2010).

51. A. A. Kamshilin et al., "Variability of microcirculation detected by blood pulsation imaging," PLoS One 8(2), e57117 (2013).

52. L. T. Pearson et al., "Effect of adding tetracaine to bupivacaine on duration of analgesia in supraclavicular brachial plexus nerve blocks for ambulatory shoulder surgery," Proc. (Bayl. Univ. Med. Cent) 28(3), 307-311 (2015).

53. U. Rubins et al., "The analysis of blood flow changes under local anesthetic input using non-contact technique," in 3rd Int. Conf. on Biomedical Engineering and Informatics, pp. 601-604, IEEE (2010). 
54. U. Rubins, J. Spigulis, and A. Miscuks, "Application of colour magnification technique for revealing skin microcirculation changes under regional anaesthetic input," Proc. SPIE 9032, 903203 (2013).

55. S. A. Landsverk et al., "Human skin microcirculation after brachial plexus block evaluated by wavelet transform of the laser Doppler flowmetry signal," Anesthesiology 105(3), 478-484 (2006).

56. S. Lehtipalo et al., "Cutaneous sympathetic vasoconstrictor reflexes for the evaluation of interscalene brachial plexus block," 44(8), 946-952 (2000).

57. K. H. W. Lange et al., "Skin temperature measured by infrared thermography after specific ultrasound-guided blocking of the musculocutaneous, radial, ulnar, and median nerves in the upper extremity," $B r$. J. Anaesth. 106, 887-895 (2011).

58. B. J. Robinson et al., "Mechanisms whereby propofol mediates peripheral vasolidation in humans: sympathoinhibition or direct vascular relaxation?," Anesthesiology 86(1), 64-72 (1997).

59. J. Sandby-Møller, T. Poulsen, and H. C. Wulf, "Epidermal thickness at different body sites: relationship to age, gender, pigmentation, blood content, skin type and smoking habits," Acta Derm. Venereol. 83(6), 410-413 (2003).

Zbignevs Marcinkevics received his $\mathrm{PhD}$ degree in cardiovascular physiology from the University of Latvia, Riga, Latvia, in 2014. He is currently an associate professor at the Department of Human and Animal Physiology, University of Latvia. His major research interests lie at the interface between cardiovascular physiology and biophotonics, focusing on the evaluation of novel noninvasive diagnostic techniques for assessment of cutaneous microcirculation and regional hemodynamics.

Uldis Rubins received his BSc degree in physics, and his MSc and $\mathrm{PhD}$ degrees in medical physics from the University of Latvia, Riga, Latvia. Currently, he is a leading researcher in the Institute of Atomic Physics and Spectroscopy, University of Latvia, Riga, Latvia. His main research focuses on imaging photoplethysmography, analysis of pulse waveform, and spectroscopy of human skin. He has authored and coauthored over 20 publications including peer reviewed journals and conference proceedings.

Janis Zaharans received his BSc degree in physics from the University of Latvia, and an MSc in intellectual robotic systems from Riga Technical University and during ERASMUS exchange program has studied in Linköping University. He has participated in multiple projects related to cardiovascular system monitoring and diagnostics by developing devices and signal processing algorithms.

Aleksejs Miscuks received the degree of medical doctor from the Latvian Academy of Medicine 1993 and the PhD degree in medicine from the University of Latvia 2012. He has over 20 years of experience in anesthesiology and intensive care. His areas of interest are regional anesthesia and invasive pain therapy. Currently, he is working as senior consultant at the Hospital of Traumatology and Orthopedics and assistant professor at the Faculty of Medicine (University of Latvia).

Evelina Urtane received her BSc degree in biology from the University of Latvia in 2014. She is continuing studies toward her MS degree in biology, human, and animal physiology. Her current research interests include physiology of blood-vessels and circulation, regional hemodynamics, techniques for skin microcirculation monitoring.

Liga Ozolina-Moll received her $\mathrm{PhD}$ in biology from the University of Latvia in 1999, and currently she is an associate professor in the Department of Human and Animal Physiology. Since 2013 she has been the head of the Department of Human and Animal Physiology at the University of Latvia. Her research interests are in physiology of metabolism and application of photonic methods for noninvasive monitoring of human anthropometric and physiological parameters. 